\title{
The Importance of Correlated Motions on the Low Barrier Rotational Potentials of Crystalline
}

\section{Molecular Gyroscopes}

\author{
Peter D. Jarowski, Kendall N. Houk, and Miguel A. Garcia-Garibay*
}

Department of Chemistry and Biochemistry University of California, Los Angeles,

California 90095-1569

mgg@chem.ucla.edu and houk@chem.ucla.edu

SUPPORTING INFORMATION

SI 1. Raw rotational energy profile data for M-1A and M-1B using constraint sets $I$ - $I V$.

\begin{tabular}{|c|c|c|c|c|c|c|c|c|}
\hline \multirow[b]{3}{*}{$\begin{array}{c}\text { Dihedral } \\
\text { Angle (deg) }\end{array}$} & \multicolumn{8}{|c|}{ Energy $(\mathrm{kcal} / \mathrm{mol})$} \\
\hline & \multicolumn{4}{|c|}{ M-1A } & \multicolumn{4}{|c|}{ M-1B } \\
\hline & $\begin{array}{c}\text { Constraint } \\
\text { Set I }\end{array}$ & $\begin{array}{c}\text { Constraint } \\
\text { Set II }\end{array}$ & $\begin{array}{c}\text { Constraint } \\
\text { Set III }\end{array}$ & $\begin{array}{c}\text { Constraint } \\
\text { Set IV }\end{array}$ & $\begin{array}{c}\text { Constraint } \\
\text { Set I }\end{array}$ & $\begin{array}{c}\text { Constraint } \\
\text { Set II }\end{array}$ & $\begin{array}{c}\text { Constraint } \\
\text { Set III }\end{array}$ & $\begin{array}{c}\text { Constraint } \\
\text { Set IV }\end{array}$ \\
\hline 0 & 3406.00 & 2910.06 & 2865.24 & 1087.76 & 3368.26 & 2903.50 & 2843.13 & 2891.95 \\
\hline 10 & 3407.12 & 2910.60 & 2865.80 & 1087.88 & 3369.19 & 2904.27 & 2843.91 & 2892.61 \\
\hline 20 & 3409.56 & 2911.95 & 2867.16 & 1088.49 & 3371.55 & 2906.26 & 2845.75 & 2894.19 \\
\hline 30 & 3413.23 & 2914.08 & 2869.22 & 1087.58 & 3375.25 & 2909.38 & 2848.54 & 2896.66 \\
\hline 40 & 3417.91 & 2916.74 & 2869.04 & 1087.92 & 3380.22 & 2913.53 & 2852.07 & 2899.79 \\
\hline 50 & 3423.44 & 2917.32 & 2869.77 & 1088.52 & 3386.57 & 2918.43 & 2855.75 & 2903.15 \\
\hline 60 & 3430.26 & 2919.30 & 2870.74 & 1089.46 & 3394.49 & 2923.10 & 2858.63 & 2905.66 \\
\hline 70 & 3438.80 & 2921.72 & 2872.16 & 1091.03 & 3404.09 & 2926.69 & 2860.27 & 2907.28 \\
\hline 80 & 3449.26 & 2924.68 & 2874.06 & 1093.21 & 3415.34 & 2927.90 & 2860.63 & 2908.07 \\
\hline 90 & 3461.72 & 2928.16 & 2876.30 & 1095.65 & 3428.05 & 2926.94 & 2860.51 & 2907.99 \\
\hline 100 & 3476.12 & 2931.84 & 2878.63 & 1098.09 & 3438.82 & 2924.38 & 2859.80 & 2906.77 \\
\hline 110 & 3489.38 & 2934.87 & 2880.66 & 1099.70 & 3398.11 & 2921.39 & 2857.81 & 2905.12 \\
\hline 120 & 3426.46 & 2919.98 & 2882.19 & 1100.35 & 3391.73 & 2918.34 & 2855.16 & 2903.04 \\
\hline 130 & 3419.70 & 2917.57 & 2871.67 & 1092.18 & 3385.52 & 2915.11 & 2852.38 & 2900.61 \\
\hline 140 & 3414.31 & 2915.26 & 2869.39 & 1090.83 & 3379.41 & 2911.57 & 2849.51 & 2897.97 \\
\hline 150 & 3410.27 & 2913.10 & 2867.37 & 1089.15 & 3374.38 & 2908.16 & 2846.78 & 2895.41 \\
\hline 160 & 3407.56 & 2911.39 & 2865.81 & 1087.61 & 3370.79 & 2905.47 & 2844.63 & 2893.38 \\
\hline 170 & 3406.16 & 2910.33 & 2864.91 & 1086.77 & 3368.76 & 2903.87 & 2843.38 & 2892.20 \\
\hline 180 & 3406.02 & 2910.06 & 2864.72 & 1086.70 & 3368.28 & 2903.50 & 2843.15 & 2891.97 \\
\hline 190 & 3407.17 & 2910.62 & 2865.35 & 1087.13 & 3369.35 & 2904.42 & 2844.05 & 2892.77 \\
\hline 200 & 3409.55 & 2911.98 & 2866.72 & 1087.46 & 3371.93 & 2906.65 & 2846.12 & 2894.59 \\
\hline 210 & 3413.07 & 2914.08 & 2868.72 & 1087.60 & 3375.95 & 2910.10 & 2849.18 & 2897.34 \\
\hline 220 & 3417.52 & 2916.71 & 2869.01 & 1087.98 & 3381.35 & 2914.59 & 2852.89 & 2900.67 \\
\hline 230 & 3422.75 & 2917.26 & 2869.69 & 1088.60 & 3388.20 & 2919.44 & 2856.31 & 2903.74 \\
\hline 240 & 3429.08 & 2919.23 & 2870.66 & 1089.64 & 3396.50 & 2923.59 & 2858.82 & 2905.87 \\
\hline 250 & 3436.89 & 2921.63 & 2872.08 & 1091.37 & 3406.12 & 2926.74 & 2860.27 & 2907.31 \\
\hline 260 & 3446.31 & 2924.58 & 2873.97 & 1093.69 & 3416.79 & 2927.89 & 2860.62 & 2908.06 \\
\hline 270 & 3457.29 & 2928.03 & 2876.22 & 1096.13 & 3427.75 & 2927.05 & 2860.53 & 2908.06 \\
\hline 280 & 3469.70 & 2931.66 & 2878.56 & 1098.31 & 3436.23 & 2924.33 & 2859.88 & 2906.90 \\
\hline 290 & 3483.25 & 2934.74 & 2880.62 & 1099.75 & 3396.72 & 2921.18 & 2857.72 & 2905.16 \\
\hline 300 & 3426.52 & 2935.88 & 2882.17 & 1100.36 & 3390.77 & 2918.08 & 2854.98 & 2902.93 \\
\hline 310 & 3419.99 & 2917.74 & 2871.85 & 1092.16 & 3384.97 & 2914.85 & 2852.21 & 2900.44 \\
\hline 320 & 3414.67 & 2915.44 & 2869.59 & 1090.79 & 3379.29 & 2911.43 & 2849.41 & 2897.89 \\
\hline 330 & 3410.57 & 2913.27 & 2867.55 & 1089.11 & 3374.48 & 2908.17 & 2846.78 & 2895.43 \\
\hline 340 & 3407.74 & 2911.51 & 2865.92 & 1087.64 & 3370.93 & 2905.55 & 2844.69 & 2893.46 \\
\hline 350 & 3406.21 & 2910.38 & 2864.95 & 1086.81 & 3368.84 & 2903.92 & 2843.41 & 2892.26 \\
\hline 360 & 3406.00 & 2910.06 & 2864.70 & 1086.67 & 3368.26 & 2903.49 & 2843.12 & 2891.94 \\
\hline
\end{tabular}

SI 2. Potential Energy Profiles and Energetic Breakdown.

The type and magnitude of the forces acting on a rotator are analyzable from the molecular mechanics calculation. The energy profiles of M-1A and M-1B using constraint set $I V$ are decomposed into their 
constituent mechanical $\left(\mathrm{E}_{\mathrm{m}}\right)$ (stretch, bend, and torsion), van der Waals or steric $\left(\mathrm{E}_{\mathrm{vdw}}\right)$, and electrostatic $\left(E_{e}\right)$ energies. The results are shown below.
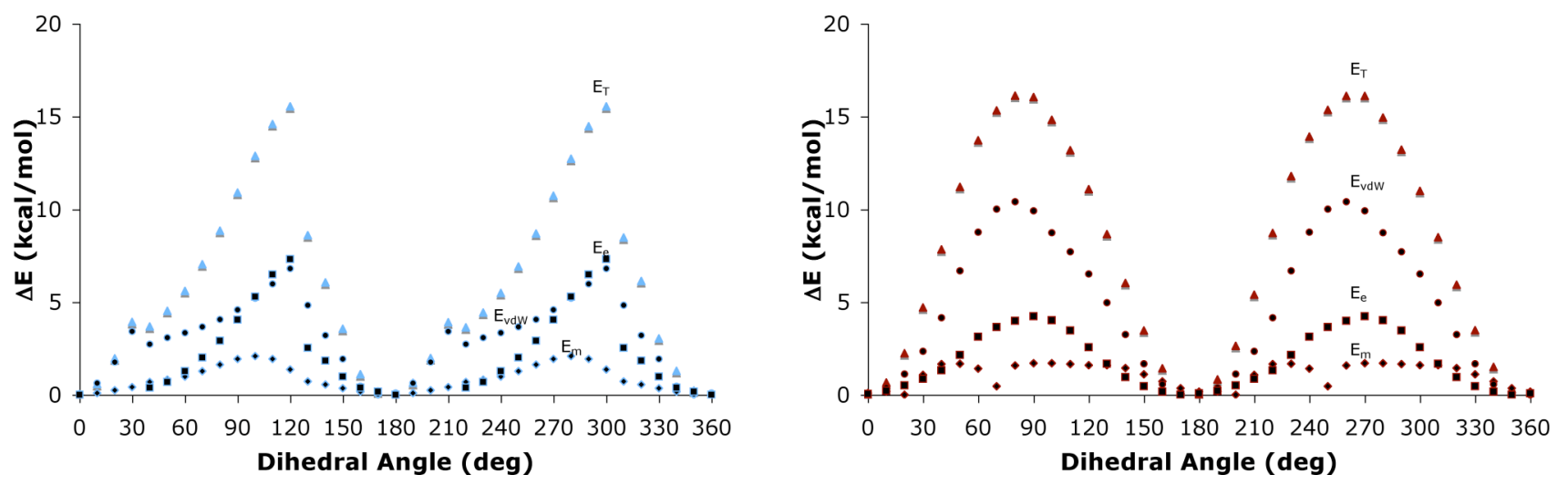

Caption: Rotational potential energy profiles (triangles) and energetic breakdown into the van der Waals $\left(\mathrm{E}_{\mathrm{vdW}}\right)$ (circles), electrostatic $\left(\mathrm{E}_{\mathrm{e}}\right)$ (squares), and mechanical (sum of the stretch, bend, and torsion strain terms) $\left(\mathrm{E}_{\mathrm{m}}\right)$ (diamonds) for the clathrate $\mathbf{M - 1 A}$ (left) and desolvated $\mathbf{M - 1 B}$ (right) rotors using constraint set IV.

The $\mathrm{E}_{\mathrm{vdw}}$ and $\mathrm{E}_{\mathrm{e}}$ contributions to the rotational energy profile for $\mathbf{M - 1 A}$ are close in magnitude, and they are both far greater than $\mathrm{E}_{\mathrm{m}}$. It should be noted that the electrostatic contribution in aromatic systems arise from the quadrupolar nature of their structures, with electrostatic potentials that vary from positive in the center of the ring, to negative on the edges. The $\mathrm{E}_{\mathrm{vdw}}$ and $\mathrm{E}_{\mathrm{e}}$ terms at the maxima account for 44 and $47 \%$ of the barrier height, respectively. The shapes of these traces are very different. The $\mathrm{E}_{\mathrm{e}}$ trace climbs nearly exponentially from the minimum to the maximum and then drops off in a similar fashion. The $\mathrm{E}_{\mathrm{vdw}}$ trace is non-monotonic as there is a slight kink in the profile occurring at 30 and $210^{\circ}$. The same kink is found in the overall trace. The $\mathrm{E}_{\mathrm{m}}$ contribution has a nearly sinusoidal trace with a maximum that is displaced by $-20^{\circ}$ from the overall maxima. The abrupt decrease in energy after the maxima and the kink in the profile just described may be the outcome of hysteresis, i.e. the profile may not represent the lowest energy pathway to rotation. Indeed, if the profile is obtained by rotation in the opposite direction the maxima shift to 70 and $250^{\circ}$ with barrier heights of about $20 \mathrm{kcal} / \mathrm{mol}$, as described in the computational method section. However molecular dynamics runs at the minimum and 
maximum of this profile did not locate a lower energy structure than was found from the dihedral drive as shown.

A similar analysis of $\mathbf{M - 1 B}$ reveals a barrier that is $65 \% \mathrm{E}_{\mathrm{vdw}}$ and $21 \% \mathrm{E}_{\mathrm{e}}$. Thus, the $\mathbf{M - 1 B}$ has a larger steric contribution for the barrier and a smaller electrostatic contribution compared to M-1A. The similar magnitudes of these energy terms for M-1A thus results from a relatively low steric contribution and a slightly larger electrostatic contribution illuminated by comparison to the case of M-1B. Although the steric contribution dominates the profile in this case, the electrostatic contribution is still larger than the mechanical one. All the traces, but that of $E_{m}$ are essentially sinusoidal, and the maximum of $E_{e}$ is displaced by $10^{\circ}$ with respect to the overall maximum.

SI 3. Frequency $(v)$ of data points versus deviation $(\AA)$ of the calculated equilibrium structure at $0^{\circ}$ dihedral angle from the X-ray structure for M-1A (blue) and M-1B (red). The frequency data is collected in data ranges of $0.1 \AA$.

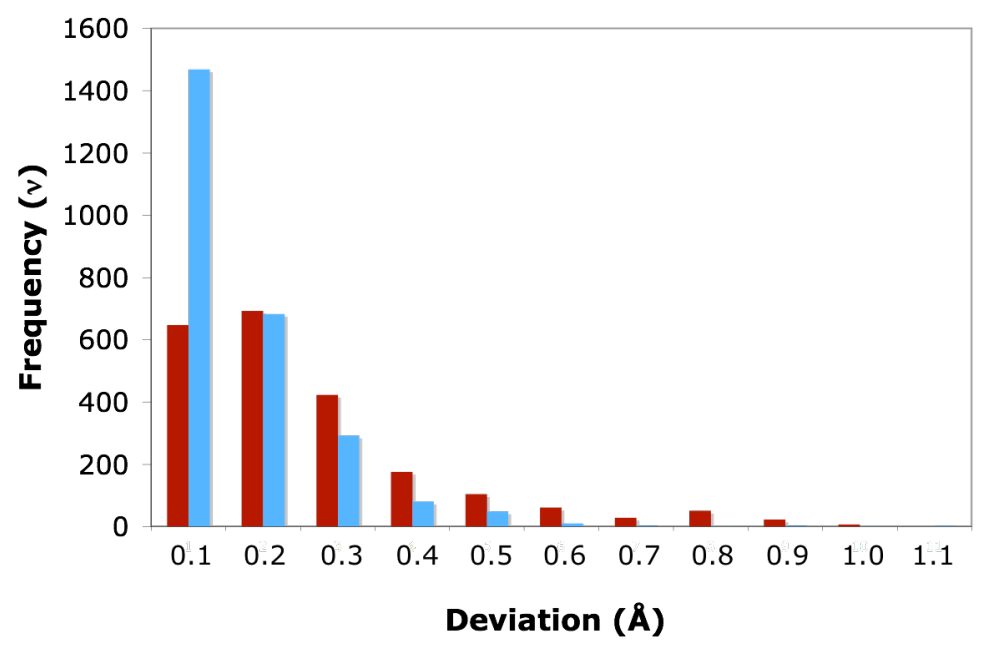

SI 4. Deviation $(\AA)$ from calculated $0^{\circ}$ equilibrium structure at $360^{\circ}$ dihedral angle versus distance $(\AA)$ from the center-of-mass of the models M-1A (blue squares) and M-1B (red circles). 


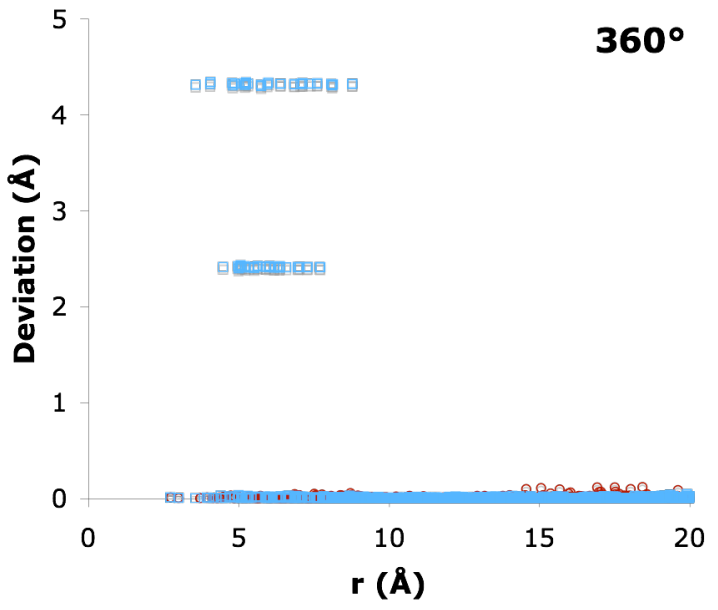

